\title{
Analysis on imaging diagnostic value of bone tumor
}

\author{
Rui Jiang ${ }^{1}$, Ziyan Zhang ${ }^{2}$, Han $\mathrm{Wu}^{1}$ and Chao Zhang ${ }^{3,}$ a, * \\ ${ }^{1}$ Department of orthopedics, China-Japan Union Hospital of Jilin University, Jilin 130033, China \\ ${ }^{2}$ Department of orthopedics, The Second Hospital of Jilin University, Jilin 130041, China \\ ${ }^{3}$ Department of ophthalmology, The Second Hospital of Jilin University, Jilin 130041, China \\ azhangchao0701@163.com
}

Keywords. Digital X-radiography, computed tomography, magnetic resonance imaging, bone tumor, clinical value.

\begin{abstract}
Objective: to discuss the clinical value of digital X-radiography (DR), computed tomography (CT) and magnetic resonance imaging (MRI) in diagnosing bone tumors; Methods: 45 bone tumor patients accepted from November 2010 to February 2013 were retrospectively analyzed. All 45 patients underwent DR examination, wherein 35 cases underwent CT examination and 21 cases underwent MRI examination. Results: DR and CT are similar in displaying bone tumor bone destruction, hyperplasia, and sclerosis and lesion border. However, DR is not as prominent as CT in displaying spine, basis cranii and pelvic lesions, and CT is more prominent for tiny lesion as thin as pin-point. In addition, it is more prominent than DR in displaying soft tissue shadow. DR is the best in displaying periosteal reaction, MRI is the most specific in displaying bone marrow edema and soft tissue giant tumor shadow, and it is the worst in the aspect of displaying bone destruction, hyperplasia, sclerosis, calcified plaque, etc. Conclusion: clinical diagnosis of bone tumor is still based on DR, CT can display tumor scope and fine structure, MRI has advantages in displaying soft tissue condition and spinal cord edema. Disease condition should be combined clinically for selecting diagnosis mode.
\end{abstract}

\section{Introduction}

Imaging diagnostic methods of bone tumors include digital X -radiography (DR), computed tomography (CT) and magnetic resonance imaging (MRI). Computed tomography is adopted for CT to obtain more information of diseased region with the development of radiological technology, the lesion condition of bone tumor has excellent display effect, MRI has high organizational resolution, multiparameter imaging, no bone artifacts, no radiation damage and other advantages [1]. We compared the difference of DR, CT and MRI in the aspect of diagnosing bone tumor. It is hereby reported as follows.

\section{Data and methods}

\subsection{General data}

45 bone tumor patients accepted from November 2010 to February 2013 were retrospectively analyzed, including 31 male cases, 14 female cases, they were 12 to 69 years old with average age of (44. $1+-2.8$ ) years old. All patients were diagnosed pathologically, including 7 cases with myeloma, 4 cases with osteochondroma, 5 cases with osteosarcoma, 3 cases with non-ossifying fibroma, 5 cases with bone lipoma, 5 cases with metastatic tumor, 2 cases with fibroma, 4 cases with chondroblastoma and 10 cases with other tumors.

\subsection{Research method}

Siemens Aristos VX Pius X-ray machine was adopted for DR, its size was $35 \mathrm{~cm} * 43 \mathrm{~cm}, 35 \mathrm{~cm} *$ $35 \mathrm{~cm}$ and $25 \mathrm{~cm} * 30 \mathrm{~cm}, 45$ patients underwent DR examination. 35 patients in CT group selected Siemens Avanto Brightspeed VCT scanning, scanning voltage was 110 to $130 \mathrm{kV}$, collimation $0.5 * 8$, DFOV 300mm, reconstruction layer thickness was 5 to $10 \mathrm{~mm}$, preset value was $30 \mathrm{MA}$, screw pitch was $1.5,0.55 \mathrm{~s} /$ ring, routine soft tissue window and bone window reconstruction were carried out. 21 
patients in MRI group selected Siemens Avanto I-Class 1. 5T superconducting magnetic resonance imaging scanner, body phased array coils were used for plain scanning, general sequence was collected under quiet breathing, rapid sequence was collected under breathholding, SE sequencing T1W1, T2W1 and STIR were scanned conventionally for imaging at axial, vector and coronal directions. The layer thickness was 5 to $8 \mathrm{~mm}$, interlayer spacing was 0.5 to $1 \mathrm{~mm}$, T1 weight was SETR 480 to $490 \mathrm{~ms}$, TE14ms belongs to spin echo imaging, quick small imaging incentive imaging was FLASHTR 138ms and TE6. 2ms; T2 weight was TSETR3200 to $4000 \mathrm{~ms}$ and TE102 ms. HASTE TR 2000 to 2200 msTE60 ms was adopted for scanning.

\subsection{Statistical method}

SPSS 13. 0 software was adopted for data analysis. count data underwent 2 test, and difference of $\mathrm{P}<0.05$ had statistical significance.

\section{Result}

DR and CT are similar in displaying bone tumor bone destruction, hyperplasia, sclerosis and lesion border. However, DR is not as prominent as CT in displaying spine, basis cranii and pelvic lesions, $\mathrm{CT}$ is more prominent for tiny lesion as thin as pin-point. In addition, $\mathrm{CT}$ is more prominent than DR in displaying soft tissue shadow. However, DR is the best in displaying periosteal reaction, MRI is the most specific in displaying bone marrow edema and soft tissue giant tumor shadow. It is prominent better than DR and CT, but it has the worst performance in the aspects of displaying bone destruction, hyperplasia , sclerosis, calcified plaque, etc. Bone tumor morbidity was $2 \%$ to $3 \%$ [2] with increase trend. DR is an examination method displaying the highest skeletal system specificity at present [3]. It has the advantages of high spatial resolution, which can clearly display thin periosteal reaction, most bone changes due to bone tumors and calcification with different size in most lesions. The bone tumor has excellent stereoscopic sensation and low price. It has the disadvantages that lesion tissues with complicated dissection can not be easily diagnosed, including tumors in pelvis, skull, etc. It also has insufficient display of soft tissue shadow [4]. However, CT can fill the defect. CT can accurately display bone destruction, hyperplasia, sclerosis, calcification and other lesion border. Even smaller tumors in spine, basis cranii, pelvis and other sites with complicated structures can be clearly displayed [5], soft tissue shadow also can be clearly displayed. However, it is not as excellent as DR in displaying periosteal reaction. MRI relies on extremely high soft tissue resolution, any section imaging and other advantages for standing out in bone tumor diagnosis with imaging development, it can clearly display the relationship between tumor and surrounding neighboring tissue organs as well as corresponding bone marrow edema [6], but is not as excellent as DR in displaying calcification and skeleton changes. Clinical diagnosis of bone tumor still should be based on DR, CT can display tumor scope and fine structure. MRI has advantages in displaying soft tissue condition and spinal cord edema. Disease conditions should be combined clinically for selecting diagnosis mode [7].

\section{Discussion}

Bone tumor has complicated imaging performance, it is difficult to identify benign and malignant tumors, the principles of combining clinical manifestation, pathological examination and imaging features must be followed, thereby improving bone tumor diagnosis accuracy and reducing misdiagnosis. X -ray plain film can reflect tumor site, scope, growth mode, growth process and the relationship between tumor and surrounding tissues; It also can reflect the type of tumor bone destruction, distinguish benign tumor and malignant tumor, predict tumor components and tumor histological category, observe tumor development condition, recurrence and transference, reaction on treatment, etc. [ 1] Though X -ray plain film is simple and economic, it is the primary means for bone tumor diagnosis. However, since $\mathrm{X}$-ray plain film compresses body stereochemical structure into plane, many organization structures are overlapped to form images, and therefore its positioning value is far higher than the quantitative value. In addition, since X -ray plain film is better than CT and magnetic resonance imaging (MRI) in the aspect of density resolution, it is worse than CT and MRI in 
reflecting tumor structure definition and reflecting tumor scope in medullary space. X -ray plain film is the most basic examination method in imaging examination, and beguine and malignant tumors can be basically judged. Some tumors, such as osteochondroma, can be diagnosed by X -ray plain films. Malignant bone tumor has the following characteristics frequently: osteolytic lesion is manifested as entomophaga, sieve pore and patchy destruction. The form is irregular with fuzzy edges; the bone cortex is thin and interrupted, which disappears or produces irregular residual sclerite. The sign is an important basis to judge early malignant bone tumor; periosteal reaction shows linear, single-layer or double-layer, onion skin or vertical bone cortex high density shadow. Patients with osteosarcoma can form codman triangle; patchy and flocculent bone sclerosis, needle-like, radiated and coarse ennomoclone or thin point-shaped, strip-shaped or arch calcification shadow chondroma can be discovered in tumor failure zone or soft tissues around the tumor. Benign tumor is manifested as round or oval expansible bone destruction zone. The boundary is clear with uneven density. There is a sclerosis belt with increased density on the edge. It is thinned in expansible bone cortex. Interruption, disappearance and other similar signs appear rarely. However, tiny bone cortex destruction on common X -ray plain film can not be judged easily, and there are rare periosteal reaction and soft tissue giant tumors. $\mathrm{X}$-ray plain film has the following disadvantages: tumors in spine, basis cranii, pelvis and other sites with complicated structures has difficult $X$-ray plain film display due to organ interference. Other image examination methods should be selected aiming at the tumors in the sites.

CT scanning has prominent advantages to bone tumor diagnosis compared with $\mathrm{X}$-ray plain films, CT images can better display the erosion damage of tumors to bone cortex, medullary space and other sites, tumor-based soft tissue giant tumor formed by tumor extruding from the cortex and other performance compared with plain film more clearly and early. Lesion anatomical position, scope and adjacent structure can be displayed by $\mathrm{X}$-ray plain film more sufficiently, such as relationship with muscle, organs, blood vessels, and nerves. Since the density resolution is high, the window width and window position also can be adjusted, therefore invasion of the bone tumor in the bone, spreading scope and software package tissue lump appearance outlines can be observed at the same time. Computer program should be used for measuring and analyzing tumor size and CT value. tumor position and scope prompted by X -ray plain film are not accurate sometimes. CT can be used for adjusting the scanning scope at any time according to demands. The observation surface can be expanded, focus, distal multiple focus and metastasis focus can be traced. In addition, enhanced scanning method is adopted to improve the density different of tumor to normal tissues. Tumor diagnosis is further judged through bump enhancing category. It is another feature that CT examination is better than $\mathrm{X}$-ray plain film. Tumor tissue enhancing is closely related to tumor vascular anomaly hyperplasia and blood flow increase. Therefore, various enhancing performances after tumor enhancing can reflect tumor matrix, mass edge shape, blood supply, whether there is false envelope and interval or not, etc. from another perspective. It is helpful to identify and diagnose benign and malignant tumors, and qualitatively diagnose tumors. CT has high density resolution, which is more sensitive to bone destruction scope, the relationship between tumor and surrounding tissues as well as display of calcification and ossification [2]. CT scanning has wider bone destruction display scope, the infiltration in the medullary space is far, and a lot of low-density adipose tissues in medullary space are replaced by tumor tissues. General tumor CT value is always $>15 \mathrm{Hu}$, which can be increased to 20 to $40 \mathrm{Hu}$ and can reach more than $100 \mathrm{Hu}$ aiming at patients with ennomoclone, the fat marrow scope lasts from -100 to $-120 \mathrm{Hu}$, and a distinct density difference is formed between the tumor and normal bone marrow tissues. It is conducive to accurately define the scope of tumor. The enhanced CT scanning shows nonuniform enhancement of tumor tissues. The central area quasi-circular necrosis is free from enhanced zone. Contrast-enhanced CT three-dimensional reconstruction is sometimes more sensitive than MRI in displaying benign and malignant bone tumor and soft tissue tumor vascular nerve [3]. CT scanning has the disadvantages that its spatial resolution is not as good as normal X-ray examination, and it is obvious that tiny 1 periosteal reaction is not as clear as X-ray plain film. X -ray plain film and CT examination are fully utilized to improve the accuracy of bone tumor imaging diagnosis. In the study, CT and M RI characteristic manifestation and 
diagnostic value of malignant bone tumor tissue mass are analyzed. Patients undergo CT and M RI examination. It is discovered in the study that CT and M RI can obtain certain diagnosis effect, wherein CT can display bone change and lesion border condition more clearly and accurately. It has higher organizational resolution. Compared with CT diagnosis, M RI examination can be applied for observing the tissue form as a whole comprehensively. The components of tissues in the mass can be clearly displayed. MRI is a main examination method to diagnosis orthopedic diseases. Compared with X-ray and CT examination, MRI has higher soft tissue resolution in the aspects of diagnosing brain and spinal cord. Scanning images at the directions of cross-section, coronal, sagittal view and other directions can be obtained. MRI examination can clearly display the lesion center, lesion edge and attached tuberculousis bone destruction. T1W1 shows hypointensity, and T2W1 shows hyperintensity. Lesion scope, size, form, surrounding organ and tissue lesion conditions can be displayed clearly through MRI examination aiming at subperiosteal tuberculousis patients. T1W1 shows hypointensity, and T2W1 shows hyperintensity.MRI examination can be applied for making clear whether there is edema around the tumor or not in bone tumor patients. The lesion can be clearly displayed by T1W1 and T2W1 weight imaging as well as multi-directional imaging. The study shows that MRI examination diagnosis accuracy is higher in bone tuberculosis and bone tumor diagnosis, which is up to higher than $90 \%$. Therefore, it is obvious that bone tuberculosis and bone tumor through can be effectively identified through MRI examination. However, the examination method also has certain defects with low specificity. The calcification focus detection rate is lower. In MRI examination, invasion scope of malignant bone tumor can be clearly displayed through T1W1 and T2W1 weight imaging. However, t1W1 signal strength is easily affected by tissue blood perfusion, cell density and $\mathrm{t} 2$ penetration effect. When the $\mathrm{T} 2$ value is increased, false positive phenomena may appear, benign tumor and malignant tumor may form cytotoxic edema and hemorrhage necrosis, thereby leading to overlapping phenomena of T1W1 signal strength. The specificity is not high as a result. Pathological examination results should be combined for diagnosis. The research results are analyzed. It is clear that M RI examination diagnosis coincidence rate is $96.67 \%$, which is significantly higher than $80.00 \%$ of CT diagnosis. It is prompted that MRI examination effect is more exact with high accuracy in the aspect of diagnosis malignant bone tumor tissue mass. Meanwhile, the research shows that soft tissue benign mass and malignant mass do not have prominent difference in the aspects of scope, signal unevenness and edge fuzziness according to MRI examination. However, soft tissue benign mass and malignant mass are prominently different in the aspects of surrounding edema and invasion. It is prompted that the clinical doctors should focus on observing mass invasion and edema around the focus during identification and diagnosis on patients. The above research results are summarized, it is concluded that CT and M RI examinations are adopted for malignant bone tumor tissue mass patients with certain diagnosis effect. However, the effect of MRI examination is better, thereby providing reference for clinical doctors during diagnosis and analysis of disease condition.

\section{Acknowledgments}

This work was supported by the study of c-Myc and mTOR inhibitors in the combined targeting therapy of osteosarcoma. Jilin provincial finance department (No 3D517P373430).

\section{References}

[1]. Wu Hongying, Liang Huimin, Zeng Xiangjie. Application of CT window technique in diagnosis of malignant bone tumor. Journal of Clinical Radiology, 2001, 20 (2):132-133.

[2]. Lu Renyi. Bone tumor X -ray plain film and CT diagnosis . China Bone Tumor Bone Disease ,2002, 1 (4):209-212. 
[3]. M ori Y, Fujii M , Akisue T, et al .Three-dimensional images ofcontrast-enhanced MDCT for preoperative assessment of mus-culoskeletalmasses:comparison with MRI and plain radio-graphs.Radiat Med, 2005 (6):398-406.

[4]. Luo Zhendong, Chen Weiguo, Jia Ming, etc. Analysis on imaging performance of ilium primary malignant bone tumor. Journal of Clinical Radiology , 2011, 30 (10): 1492-1496.

[5]. Na Shengbo, Zhao Wen, Song Yanqi, etc. Characteristic comparison of CT and MRI diagnosis of malignant bone tumor soft tissue mass, 2016, 10 (3): 51-52.

[6]. Zhou Shouguo, Zhao Xiaomei, Fan Changshu, etc. Imaging diagnosis on ilium primary malignant tumor (attached with analysis on 20 cases). China CT and MRI Magazine, 2011, 9 (1): 64-66.

[7]. Zou Fei, Yang Jianfei, Tang Min, etc. Analysis on imaging performance of primary bone lymphoma. Journal of Medical Imaging, 2012, 22 (6): 1000-1003, 1006. 